\section{A PHASE 2, MULTI-ARM STUDY OF ANTI-CD47 ANTIBODY, MAGROLIMAB, IN COMBINATION WITH DOCETAXEL IN PATIENTS WITH LOCALLY ADVANCED OR METASTATIC SOLID TUMORS}

'Vivek Subbiah*, ${ }^{2}$ Ulka Vaishampayan, ${ }^{3}$ Sonam Puri, ${ }^{4}$ Lanjia Lin, ${ }^{4}$ Mark Chao, ${ }^{4}$ Giri Ramsingh, ${ }^{5}$ Shivaani Kummar, ${ }^{6} J a m e s$ Strauss, ${ }^{7}$ Sandip Patel. ${ }^{1}$ The University of Texas MD Anderson Cancer Center, Houston, TX, USA, Houston, TX, USA; ${ }^{2}$ Rogel Cancer Center, University of Michigan, Ann Arbor, MI, USA, Ann Arbor, MI, USA; ${ }^{3}$ Huntsman Cancer Center at the University of Utah, Salt Lake City, UT, USA, Salt Lake City, UT, USA; ${ }^{4}$ Gilead Sciences, Inc., Foster City, CA, USA, Foster City, CA, USA; ${ }^{5}$ Oregon Health and Science University, Portland, OR, USA, Portland, OR, USA; ${ }^{6}$ Mary Crowley Cancer Research, Dallas, TX, USA, Dallas, TX, USA; ' University of California, San Diego, San Diego, CA, USA, La Jolla, CA, USA

Background Patients with solid tumors who progress on standard chemotherapy and/or immune checkpoint inhibitors, have limited efficacy with existing standard of care chemotherapy options (objective response rates [ORR] $\sim 10 \%$ ). These patients have a significant unmet medical need. Novel agents that can safely enhance treatment efficacy are urgently needed. Magrolimab is a first-in-class monoclonal antibody that blocks the macrophage inhibitory immune checkpoint CD47, a "do not eat me" signal overexpressed on tumor cells. Pre-clinical studies provide compelling evidence that magrolimab triggers phagocytosis and eliminates cancer cells from human solid tumors and hematologic malignancies. Magrolimab has demonstrated clinical activity in both hematologic and solid tumor malignancies. Chemotherapeutic agents, including taxanes, enhance prophagocytic signals on tumor cells, leading to synergistic antitumor activity when combined with magrolimab. This study (NCT04827576) is evaluating the safety, tolerability, and efficacy of magrolimab with docetaxel in relapsed/ refractory $(\mathrm{R} / \mathrm{R})$ metastatic non-small cell lung cancer (mNSCLC), urothelial cancer (mUC), and small cell lung cancer (mSCLC).

Methods This phase 2, open-label, multi-arm study consists of a safety run-in cohort and a phase 2 cohort. Eligible patients are $\geq 18$ years old with chemotherapy and/or immunotherapy refractory mNSCLC, mSCLC, or mUC. Magrolimab is administered intravenously (IV) with an initial $1 \mathrm{mg} / \mathrm{kg}$ priming dose to mitigate on target anemia, followed by $30 \mathrm{mg} / \mathrm{kg}$ dose during cycle 1 (cycles are 21 days) in the safety run-in to identify any dose-limiting toxicities (DLTs) and determine a recommended phase 2 dose (RP2D). De-escalation may occur for DLTs per protocol. In phase 2 , following the priming dose on day 1, magrolimab RP2D will be administered on days 8 and 15 of cycle 1 ; days $1,8,15$ of cycle 2 ; and day 1 for cycles 3 and beyond. Docetaxel $75 \mathrm{mg} / \mathrm{m} 2$ (IV) is administered on day 1 of each cycle for all study participants. Patients may continue treatment until unacceptable toxicity, progressive disease by RECIST 1.1, or patient/investigator choice to discontinue. The primary endpoints are incidence of adverse events (safety and phase 2 cohorts) and ORR (phase 2). Secondary endpoints (phase 2) are progression-free survival, duration of response, and overall survival. Exploratory endpoints are to evaluate the pharmacodynamic, mechanism of action, and/or therapeutic response of biomarkers in blood and tumor biopsy samples and to explore biomarkers that may predict response to therapy. Planned enrollment is approximately 116 patients, and recruitment is ongoing.

Acknowledgements Funding provided by Gilead Sciences, Inc.

Trial Registration NCT04827576

Ethics Approval The study protocol was approved by an institutional review board before enrollment of patients.
Consent Patients provided written informed consent based on Declaration of Helsinki principles.

http://dx.doi.org/10.1136/jitc-2021-SITC2021.524 